\title{
Calculation of the quark-quark contribution to the Drell-Yan process. II. Identical-particle effects
}

\author{
A. N. Schellekens \\ Institute for Theoretical Physics, Nijmegen, The Netherlands \\ W. L. van Neerven \\ Centre Européen de Recherches Nucléaires, Geneva, Switzerland \\ (Received 17 April 1980)
}

The modification of the quark-quark contribution to lepton-pair production due to identical-quark scattering is calculated. The effect of the extra terms is found to be small.

\section{INTRODUCTION}

In a previous paper ${ }^{1}$ (see also Ref. 2) we have calculated the finite terms of the quark-quark contribution to lepton-pair production in hadron-hadron scattering. These terms remain after the factorization $^{3}$ of the mass singularities and their effect is a correction to the Drell-Yan formula. ${ }^{4}$ In Refs. 1 and 2, only the case of nonidentical quarks was considered. To obtain the complete quark-quark correction one has to add to the results of Ref. 1 the extra contribution due to identical-particle scattering. These new contributions consist of the interference terms between diagrams with and without crossed lines (the squares of the "crossed" and "noncrossed" diagrams, multiplied with a factor $\frac{1}{2}$ to account for the identical-particle final state, add up to the contributions already calculated in Ref. 1). These interference terms will be the subject of this paper. Whereas in the previous paper only an estimate of the quark-quark contribution could be given, the addition of our new results allows a precise calculation of this contribution.

The effect of the crossed terms turns out to be much smaller than the terms which are already calculated, and we find that the results of Ref. 1 remain unchanged, even quantitatively. Although we have only compared the differential cross sections $d \sigma / d Q^{2}$, this result indicates that identicalparticle contributions may be safely neglected also for cross sections which are more differential, such as $d \sigma / d Q^{2} d q_{\perp}$.

A few reasons for the smallness of the identicalparticle effects can be given $a$ priori. First of all, the diagrams under consideration are all nonplanar, and these are known to be suppressed in the large $-N$ limit compared to the planar diagrams calculated in Ref. 1. In this case, this suppression factor is $1 / N$ for an $S U(N)$ gauge theory. A second reason for the smallness of these diagrams is related to the mass singularities. As is shown in
Refs. 1 and 2 these $\log \left(p^{2} / Q^{2}\right)$ singularities are linked to $\log (1-\tau)$ terms, which determine the behavior of the finite terms in the limit $\tau-1$. This property, which is shared by all Drell-Yan corrections ${ }^{5}$ calculated up to now, reveals itself also in our new results. Since, as a simple analysis shows, the diagrams we consider have only a single-logarithmic mass singularity, one can directly conclude that the finite correction terms have only a single-logarithmic behavior near $\tau=1$, in contrast to the double logarithms we found in Ref. 1.

The most important reason for the suppression is, however, the fact that the factors in front of the aforementioned logarithms behave as $(1-\tau)^{5}$ for $\tau \rightarrow 1$. This power is much larger than could be expected.

Another aspect of the mass singularities considered here is worth mentioning. In terms of Altarelli-Parisi transition functions, ${ }^{6}$ they correspond to a transition from a quark to its own antiquark, but not to all other antiquark flavors. Therefore, these mass singularities violate the flavor-singlet character of the sea by reflecting the nonsinglet character of a hadron into the sea distributions. In Ref. 7 the mass singularities were investigated for this reason, by means of the operator-productexpansion method.

The results of the lepton-pair production diagrams will be presented in Sec. II. As in Ref. 1 the corresponding deep-inelastic-scattering diagrams have to be calculated for the subtraction of the mass singularities, if deep-inelastic-scattering processes are used to define and measure the distribution functions. This calculation will be discussed in Sec. III.

Finally, in Sec. IV, these results will be combined. These calculations were done using a method which proved to be successful for the nonidentical-particle contributions and was briefly discussed in Ref. 1. This method makes an extensive use of SCHOONSCHIP, ${ }^{8}$ not only for trace cal- 
culations but also for the four-dimensional phasespace integral. The major part of the programs used was already tested thoroughly in our previous calculation. Furthermore, all integrations have been tested numerically.

\section{THE LEPTON-PAIR PRODUCTION DIAGRAMS}

These diagrams can be subdivided into two classes, drawn in Figs. 1 and 2. If the external lines are taken on shell these classes are gauge-invariant sets of diagrams. In addition to the diagrams of Figs. 1 and 2, there are of course diagrams with $p_{1}$ and $p_{2}$ interchanged.

To investigate the singular behavior of these sets one can consider the diagrams with double propagators. Each set contains one diagram of that type, namely, the first diagram of Fig. 1 and the fourth diagram of Fig. 2.

The double denominator in the first set has a singularity, but the one in the second set is finite. From this fact one may conclude that only the first set will give mass singularities. In an arbitrary gauge these mass singularities cannot however be attributed completely to the double-propagator diagram, but they will also get contributions from the other three diagrams. Only in a physical gauge is the entire singularity due to the first diag ram.

In Ref. 1 two methods were discussed to regularize the mass singularities. One of these, the onshell method, is to use particle masses as the regularization parameter and to take all the ingoing and outgoing fermion lines on shell. The other one, the off-shell method, is to neglect all masses and to take the incoming fermions off shell. In fact, it turns out to be sufficient to take only one of these fermion lines off shell, namely the line with momentum $p_{1}$ in Fig. 1. This second method will be used in this paper. The final result will be independent of this choice, as we have
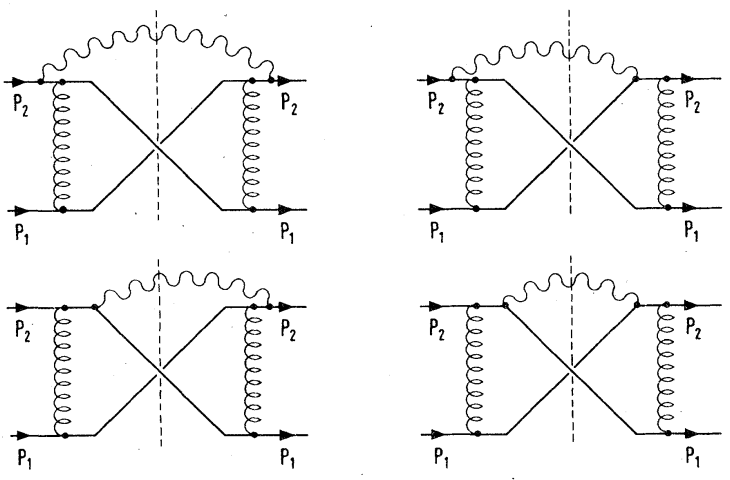

FIG. 1. Class of lepton-pair production diagrams contributing to the mass singularity. Curly lines represent gluons; wavy lines photons.
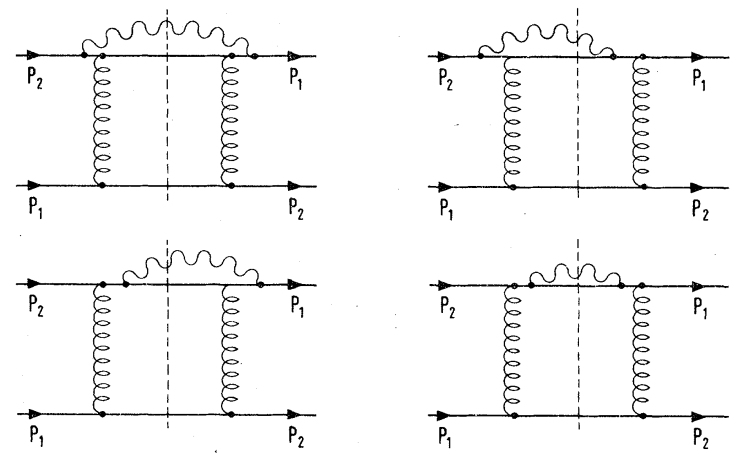

FIG. 2. Class of nonsingular lepton-pair production diagrams. (Notice that the ingoing momenta are interchanged in the right half of the diagram.)

checked explicitly for the results of Ref. 1. With $p_{2}$ on shell the results will also be independent of the gauge choice, and all gauge dependence will cancel separately in the lepton-pair production and the deep-inelastic-scattering contribution. (In general only the final result, obtained by subtraction of the deep-inelastic-scattering result, will be gauge-independent.) For our calculations we used the Feynman gauge.

The complete quark-quark cross section consists of the following factors:

$$
\begin{aligned}
\frac{d \sigma_{c r}^{\hat{q} q}}{d Q^{2}}=\frac{1}{4} \frac{1}{N^{2}} F_{c}(N) e_{q}{ }^{2} e^{2} g^{4}\left(\frac{e^{2}}{6 \pi Q^{2}}\right)[(-1)(1 / 2)(2)] \\
\times \frac{1}{2 s} \int \frac{d^{3} p_{3}}{(2 \pi)^{3} 2 E_{3}} \frac{d^{3} p_{4}}{(2 \pi)^{3} 2 E_{4}} \frac{d^{4} q}{(2 \pi)^{4}} \delta^{4}\left(q^{2}-Q^{2}\right) 2 \pi^{4} \\
\quad \times \delta^{4}\left(p_{1}+p_{2}-p_{3}-p_{4}-q\right) \\
\times\left[-g^{\mu \nu} M_{\mu \nu}^{1}\left(p_{1}\right)-g^{\mu \nu} M_{\mu \nu}^{1}\left(p_{2}\right)-2 g^{\mu \nu} M_{\mu \nu}^{2}\right] .
\end{aligned}
$$

The factors are, respectively, a spin- and coloraveraging factor and a color factor; $e_{q}$ is the charge of the quark and $e$ and $g$ are the QED and quantum-chromodynamic coupling constants. Then there is a factor because of the lepton-pair phasespace integral, a factor $(-1)$ because of the crossed fermion lines, a factor $\frac{1}{2}$ to account for the double counting due to the identical-particle final state, a factor 2 because we are dealing with an interference contribution, a flux factor, and the usual phase-space integral. Finally, $M_{\mu \nu}^{1}$ denotes the sum of the traces of Fig. 1 and $M_{\mu \nu}^{2}$ those of Fig. 2.

The color factor is, in the case of an $\mathrm{SU}(\mathrm{N})$ gauge theory,

$$
F_{c}(N)=\sum_{a, b} \operatorname{Tr}\left(T^{a} T^{b} T^{a} T^{b}\right)=-\frac{N^{2}-1}{4 N}
$$

if the $\mathrm{SU}(N)$ generators are normalized in the following way:

$$
\operatorname{Tr}\left(T^{a} T^{b}\right)=\frac{1}{2} \delta_{a b} .
$$


This result can be compared to the color factor of the nonidentical-quark contribution:

$$
F_{c}(N)=\sum_{a, b} \operatorname{Tr}\left(T^{a} T^{b}\right) \operatorname{Tr}\left(T^{a} T^{b}\right)=\frac{N^{2}-1}{4}
$$

This shows explicitly the $1 / N$ suppression mentioned in Sec. I.

For the diagrams of Fig. 1 we find the following result:

$$
\begin{aligned}
\frac{d \sigma_{c r}^{1}\left(p_{1}\right)}{d Q^{2}}= & \frac{4 \pi \alpha^{2}}{3 \hat{s} Q^{2}}\left(\frac{\alpha_{s}}{\pi}\right)^{2} \frac{N^{2}-1}{16 N^{3}} e_{q}{ }^{2} \\
& \times\left[P(\hat{\tau}) \ln \left(\frac{-p_{1}^{2}}{Q^{2}} \frac{\hat{\tau}^{2}}{1-\hat{\tau}}\right)+R_{\mathrm{DY}}^{(1)}(\hat{\tau})\right] .
\end{aligned}
$$

Here $\hat{s}$ is the $q-q$ c.m. energy $\left(p_{1}+p_{2}\right)^{2} ; \hat{\tau}=Q^{2} / \hat{s}$. The function $R_{\mathrm{DY}}^{(1)}(\tau)$ is given in the Appendix; the function $P$ equals

$$
\begin{aligned}
P(\tau)= & \frac{1+\tau^{2}}{1+\tau}\left[\ln ^{2} \tau-4 \ln \tau \ln (1+\tau)-4 \mathrm{Li}_{2}(-\tau)-\frac{\pi^{2}}{3}\right] \\
& +2(1+\tau) \ln \tau+4(1-\tau) .
\end{aligned}
$$

The complete result of the diagrams of Fig. 2 turns out to be surprisingly simple,

$$
\frac{d \sigma_{c r}^{2}}{d Q^{2}}=\frac{4 \pi \alpha^{2}}{3 \hat{s} Q^{2}}\left(\frac{\alpha_{s}}{\pi}\right)^{2} \frac{N^{2}-1}{16 N^{3}} e_{q}^{2} R_{\mathrm{DY}}^{(2)}(\hat{\tau}),
$$

where

$$
\begin{aligned}
R_{\mathrm{DY}}^{(2)}(\tau)= & (1-\tau)^{2}\left[2 \operatorname{Li}_{3}(1-1 / \tau)-3 \mathrm{Li}_{2}(1-1 / \tau)\right] \\
& +\left(\frac{7}{2}-3 \tau\right) \ln \tau+\frac{1}{2}(1-\tau)+\frac{13}{4}(1-\tau)^{2} .
\end{aligned}
$$

The functions $P(\tau), R_{\mathrm{DY}}^{(1)}(\tau)$, and $R_{\mathrm{DY}}^{(2)}(\tau)$ have a smooth power behavior for $\tau \rightarrow 1$; all the $\ln (1-\tau)$ factors are given explicitly in (2.5). The leading behavior is

$$
\begin{aligned}
& P(\tau)=\frac{1}{10}(1-\tau)^{5}+O\left((1-\tau)^{6}\right), \\
& R_{\mathrm{D}}^{(1)}(\tau)=\left(\pi^{2} / 6-1\right)(1-\tau)^{2}+O\left((1-\tau)^{3}\right), \\
& R_{\mathrm{DY}}^{(2)}(\tau)=-\frac{2}{3}(1-\tau)^{3}+O\left((1-\tau)^{4}\right)
\end{aligned}
$$

The complete parton cross section is

$\frac{d \sigma_{c r}^{\alpha q}}{d Q^{2}}=\frac{d \sigma_{c r}^{(1)}\left(p_{1}\right)}{d Q^{2}}+\frac{d \sigma_{c r}^{(1)}\left(p_{2}\right)}{d Q^{2}}+2 \frac{d \sigma_{c r}^{(2)}}{d Q^{2}}$.

Therefore, the contribution to the hadronic cross section is

$$
\begin{aligned}
& \frac{d \sigma_{H}^{c r}}{d Q^{2}}=\frac{4 \pi \alpha^{2}}{3 Q^{4}}\left(\frac{\alpha_{s}}{\pi}\right)^{2} \frac{N^{2}-1}{16 N^{3}} e_{q}{ }^{2} \int d x_{1} d x_{2} \theta\left(x_{1} x_{2}-\tau\right) f_{q}\left(x_{1}\right) f_{q}\left(x_{2}\right) \\
& \times \hat{\tau}\left\{P(\hat{\tau})\left[\ln \left(\frac{-p_{1}{ }^{2} \hat{\tau}^{2}}{Q^{2}(1-\hat{\tau})}\right)+\ln \left(\frac{-p_{2}{ }^{2} \hat{\tau}^{2}}{Q^{2}(1-\hat{\tau})}\right)\right]+2 R_{\mathrm{DY}}^{(1)}(\hat{\tau})+2 R_{\mathrm{DY}}^{(2)}(\hat{\tau})\right\},
\end{aligned}
$$

where $\hat{\tau}=\tau / x_{1} x_{2}$.

\section{THE DEEP-INELASTIC-SCATTERING DIAGRAMS}

In this case there is only one class of diagrams, depicted in Fig. 3. To regularize the mass singularities of these diagrams, we have to use the same method as in Sec. III, which implies that the ingoing line with momentum $p_{1}$ must be off-shell, whereas the outgoing antiquark line must be on-shell, like the outgoing quark lines.

The complete expression for the contribution of these diagrams to the structure function $\nu W_{2}$ of a quark is

$$
\nu W_{2}^{q}\left(x, Q^{2}\right)=-\frac{1}{N} F_{c}(N) e_{q}^{2} g^{4} \frac{1}{4 \pi} \frac{d^{3} p_{3}}{2 E_{3}(2 \pi)^{3}} \frac{d^{3} p_{4}}{2 E_{4}(2 \pi)^{3}} \frac{d^{3} p_{2}}{2 E_{2}(2 \pi)^{3}}(2 \pi)^{4} \delta^{4}\left(P_{i}-P_{f}\right)\left[-\frac{1}{2} x\left(g^{\mu \nu}-12 \frac{x^{2}}{Q^{2}} p_{1}^{\mu} p_{1}^{\nu}\right)\right] T_{\mu \nu}
$$

The factors are already explained in Sec. II. The factor $1 / 4 \pi$ is related to our definition of $W_{\mu \nu}$ for a parton:

$$
W_{\mu \nu}^{j}=\frac{1}{4 \pi} \sum_{n}\left\langle J_{\mu}(0) \mid n\right\rangle\left\langle n\left|J_{\nu}(0)\right| j\right\rangle
$$

where $j$ denotes an (anti)quark or a gluon and $n$ any intermediate state.

When the traces and the integrals in (3.1) are calculated one arrives at the following result:

$$
\begin{aligned}
\nu W_{2}^{c r}\left(x, Q^{2}\right)= & \frac{N^{2}-1}{16 N^{2}}\left(\frac{\alpha_{s}}{\pi}\right)^{2} e_{\alpha}{ }^{2} x \\
& \times\left[P(x) \ln \left(\frac{-p_{1}{ }^{2} x^{2}}{Q^{2}}\right)+R_{\mathrm{DIS}}(x)\right] .
\end{aligned}
$$

The function $P(x)$ is defined by (2.6) and $R_{\mathrm{DIS}}(x)$ is given in the Appendix. The expansion of $R_{\mathrm{D} \text { IS }}$ near $x=1$ is as follows:

$$
R_{\mathrm{DIS}}(x)=\left(2-\frac{\pi^{2}}{6}\right)(1-x)+O\left((1-x)^{2}\right) .
$$



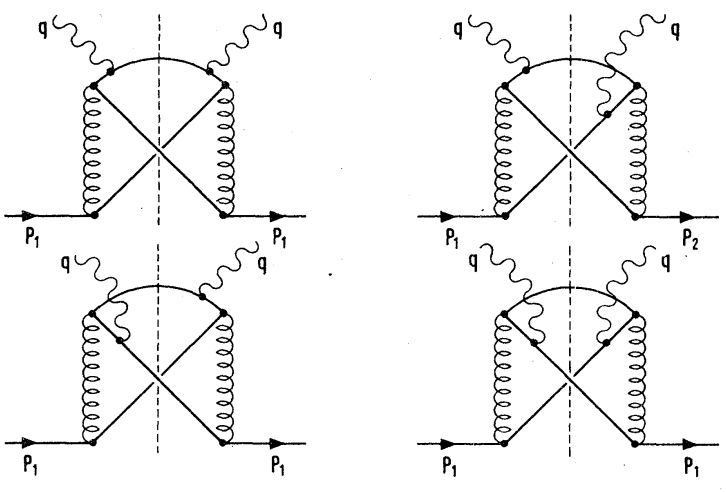

FIG. 3. Deep-inelastic-scattering crossed diagrams.

The mass singularity of (3.3) can be compared directly to the corresponding result of Ref. 7, calculated by means of the operator-product-expansion method, and Ref. 9. In the latter paper the transition function is calculated directly, and the result agrees with our result. The transition function obtained in Ref. 7, however, is a factor 4 larger than our result. ${ }^{10}$

\section{RESULTS AND CONCLUSIONS}

To be able to subtract the mass singularities in (2.5) we use (3.3) to define a "renormalized" distribution function. An important difference with the case discussed in Ref. 1 should be mentioned here. In that case the deep-inelastic-scattering mass singularities were distributed equally over a $q_{i} q_{j}$ and a $q_{i} \bar{q}_{j}$ transition, whereas in the case studied in this paper, the entire mass singularity must be attributed to a $q_{i} \bar{q}_{i}$ transition. As we have already mentioned in Sec. II, logarithmic mass singularities only originate from diagrams in Fig. 3 where both virtual photons are attached to the antiquark line, provided we are working in the axial gauge. Another argument that the mass singularities only contribute to the quark-antiquark transition function is the following. Suppose we replace the external electromagnetic current by a flavor changing one. Close inspection of the graphs in Fig. 3 reveals that two of the quarks in the final state are identical to the one in the initial state. Provided that the process exists we can argue that $q_{i} \bar{q}_{i}$ transitions always occur, whereas $q_{i} q_{j}$ transitions can only appear for certain combinations of $i$ and $j$ depending on the process which one has chosen. This would imply that the $q_{i} q_{j}$ transitions are interaction (weak, electromagnetic, etc.) dependent which is in conflict with universality of the mass singularities in the transition functions.

As an example we consider the processes $\gamma u$ $\rightarrow u u \bar{u}$ and $W^{+} u \rightarrow u u \bar{d}$. One observes immediately that in the first process $\Gamma_{u u}$ and $\Gamma_{u \bar{u}}$ are possible, whereas in the latter one $\Gamma_{u u}$ is excluded. Therefore, the deep-inelastic graphs in Fig. 3 cannot contribute to $\Gamma_{u u}$.

The factorization of the mass singularities does not uniquely fix the factorization of the nonsingular terms, because terms can be shifted arbitrarily from the cross sections to the distribution functions. For the subtraction of the singularities we will use the same convention as in Ref. 1, which means that we will absorb the entire deep-inelastic-scattering contribution in the antiquark distribution function, so that there are no finite correction terms left. Then the antiquark distribution function is modified as follows:

$$
f_{\bar{q}}\left(x, Q^{2}\right)=f_{\bar{q}}(x)+\left(\frac{\alpha_{s}}{\pi}\right)^{2} \int_{x}^{1} d \xi \frac{f_{q}(\xi)}{\xi} \frac{N^{2}-1}{16 N^{2}}\left[P(x) \ln \left(\frac{-p_{1}{ }^{2} x^{2}}{Q^{2} \xi^{2}}\right)+R_{\mathrm{D} \mathrm{IS}}\left(\frac{x}{\xi}\right)\right] .
$$

When the Drell-Yan formula is modified accordingly this yields the following extra terms:

$$
\begin{array}{rl}
\frac{d \sigma_{H}^{\text {corr }}}{d Q^{2}}=\frac{-4 \pi \alpha^{2}}{3 Q^{4}}\left(\frac{\alpha_{s}}{\pi}\right)^{2} e_{q}{ }^{2} \frac{N^{2}-1}{16 N^{3}} \int_{0}^{1} & d x_{1} d x_{2} \theta\left(x_{1} x_{2}-\tau\right) f_{q}\left(x_{1}\right) f_{q}\left(x_{2}\right) \\
& \times \hat{\tau}\left[P(\hat{\tau}) \ln \left(-\frac{p_{1}{ }^{2} \hat{\tau}^{2}}{Q^{2}}\right)+P(\hat{\tau}) \ln \left(-\frac{p_{2}{ }^{2} \hat{\tau}^{2}}{Q^{2}}\right)+2 R_{\mathrm{DIS}}(\hat{\tau})\right] .
\end{array}
$$

The final result is obtained by adding $(2.11)$ and (4.2):

$$
\frac{d \sigma_{c r}^{\alpha q}}{d Q^{2}}=\frac{4 \pi \alpha^{2}}{3 Q^{4}}\left(\frac{\alpha_{s}}{\pi}\right)^{2} e_{q}^{2} \frac{N^{2}-1}{16 N^{3}} 2 \hat{\tau} \Sigma_{c r}^{\alpha q}(\hat{\tau})
$$

with

$$
\Sigma_{c r}^{q q}=R_{\mathrm{DY}}^{(1)}(\tau)+R_{\mathrm{DY}}^{(2)}(\tau)-R_{\mathrm{DIS}}(\tau)-P(\tau) \ln (1-\tau) .
$$

We compare this with the results of Ref. 1:

$$
\begin{aligned}
\frac{d \sigma}{d Q^{2}}= & \frac{4 \pi \alpha^{2}}{3 Q^{4}}\left(\frac{\alpha_{s}}{\pi}\right)^{2} \frac{N^{2}-1}{16 N^{2}} \tau \\
& \times\left[\left(e_{1}{ }^{2}+e_{2}{ }^{2}\right) \Sigma^{\alpha q}(\tau)+2 e_{1} e_{2} \sum_{I}^{q q}(\tau)\right],
\end{aligned}
$$

for charges $e_{1}$ and $e_{2}$. In Fig. 4 we have plotted these two functions together with (4.3). The be- 


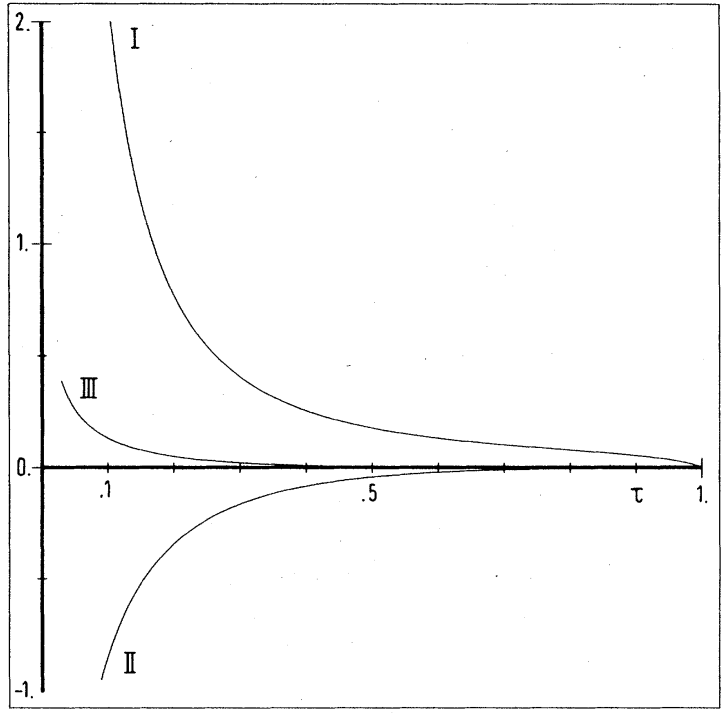

FIG. 4. Contribution of the crossed diagrams compared with the nonidentical-particle terms. Curve I represents [( $\left.\left.N^{2}-1\right) /\left(16 N^{2}\right)\right] \Sigma^{q \alpha}$, curve II: $\left[\left(N^{2}-1\right) /\left(16 N^{2}\right)\right] \Sigma_{I}^{q Q}$, and curve III: $\left[\left(N^{2}-1\right) /\left(16 N^{3}\right)\right] \Sigma_{c r}^{q Q}$. havior of the new contribution near $\tau=1$ is dominated by $R_{\mathrm{DIS}}(\tau)$ because this is the only term which is linear in this limit. ${ }^{11}$ As Fig. 4 clearly shows, the identical-particle contribution is an order of magnitude smaller than the terms already calculated. The effect on the hadron-hadron cross section is still further reduced because only part of the cross section is due to identical-particle scattering. Therefore, we may conclude that this effect can be neglected completely.

Note added. After the completion of this work we received a paper by M. Chaichian et al. ${ }^{13}$ who calculate the contribution of the $q q$ process to the transverse-momentum distribution of lepton pairs. They include the identical-particle contributions and find that their effect is small.

\section{ACKNOWLEDGMENTS}

Part of this work was included in the research program of the Stichting voor Fundamenteel Onderzoek der Materie (FOM) with financial support from the Nederlandse Organisatie voor Zuiver Wetenschappelijk Onderzoek (ZWO). One of us (W.v.N.) thanks Br. R. Petronzio for useful discussions.

\section{APPENDIX}

In this appendix we given the complete expressions for the functions $R_{\mathrm{DY}}^{(1)}(\tau)$ and $R_{\mathrm{DIS}}(\tau)$. The functions $\mathrm{Li}_{2}$ and $\mathrm{Li}_{3}$ are defined in Ref. 1 .

The functions are

$$
\begin{aligned}
R_{\mathrm{DY}}^{(1)}(\tau)= & \frac{1+\tau^{2}}{1+\tau}\left[\frac{\pi^{2}}{2} \ln \tau-\pi^{2} \ln (1+\tau)-\frac{11}{6} \ln ^{3} \tau+3 \ln (1+\tau) \ln ^{2} \tau+2 \ln ^{3}(1+\tau)-2 \ln \tau \mathrm{Li}_{2}(1-1 / \tau)-10 \mathrm{Li}_{3}(1-\tau)\right. \\
& \left.\quad-6 \mathrm{Li}_{3}(1-1 / \tau)+2 \mathrm{Li}_{3}\left(1-\tau^{2}\right)+2 \mathrm{Li}_{3}(-\tau)-12 \mathrm{Li}_{3}\left(\frac{1}{1+\tau}\right)+12 \mathrm{Li}_{3}(1)+2 \ln \tau \mathrm{Li}_{2}(-\tau)\right] \\
+ & \tau\left[10 \ln \tau-3 \ln ^{2} \tau+\frac{2}{3} \pi^{2} \ln (1+\tau)+2 \ln (1+\tau) \ln ^{2} \tau-\frac{4}{3} \ln ^{3}(1+\tau)+4 \mathrm{Li}_{3}(-\tau)+8 \mathrm{Li}_{3}\left(\frac{1}{1+\tau}\right)-4 \mathrm{Li}_{3}(1)\right] \\
+ & (1-\tau)\left(14-\frac{\pi^{2}}{6} \ln \tau+\frac{1}{6} \ln ^{3} \tau\right)-2(1+1 / \tau)\left[\ln \tau \ln (1+\tau)+\mathrm{Li}_{2}(-\tau)\right]+4 \mathrm{Li}_{2}(1-1 / \tau)+\frac{\pi^{2}}{6}(2 \tau-4)
\end{aligned}
$$

and

$$
\begin{aligned}
R_{\mathrm{DIS}}(\tau)= & \left(\frac{1+\tau^{2}}{1+\tau}\right)\left[-2 \ln \tau-\frac{\pi^{2}}{3} \ln \tau+\frac{\pi^{2}}{3} \ln (1+\tau)+3 \ln (1+\tau) \ln ^{2} \tau-\frac{2}{3} \ln ^{3}(1+\tau)+2 \mathrm{Li}_{2}(-\tau) \ln \tau\right. \\
& \left.+2 \mathrm{Li}_{3}(-\tau)+4 \mathrm{Li}_{3}\left(\frac{1}{1+\tau}\right)-2 \mathrm{Li}_{3}(1)\right] \\
& +\tau\left[4 \ln ^{2} \tau \ln (1+\tau)+\frac{4}{3} \pi^{2} \ln (1+\tau)-\frac{8}{3} \ln ^{3}(1+\tau)+8 \mathrm{Li}_{3}(-\tau)+16 \mathrm{Li}_{3}\left(\frac{1}{1+\tau}\right)-8 \mathrm{Li}_{3}(1)\right] \\
& +\left(8+6 \tau+12 \tau^{2}+2 \tau^{-1}-\frac{18}{5} \tau^{3}+\frac{2}{5} \tau^{-2}\right)\left[\mathrm{Li}_{2}(-\tau)+\ln (1+\tau) \ln \tau\right]+\ln \tau\left(-\frac{24}{5}-\frac{54}{5} \tau+\frac{18}{5} \tau^{2}-\frac{2}{5} \tau^{-1}\right) \\
& +\ln ^{2} \tau\left(-2-2 \tau-6 \tau^{2}+\frac{9}{5} \tau^{3}\right)+\frac{\pi^{2}}{6}\left(2+2 \tau+12 \tau^{2}-\frac{18}{5} \tau^{3}\right)+\frac{2}{5} \tau^{-1}+\frac{18}{5} \tau^{2}+\frac{48}{5} \tau-\frac{68}{5}
\end{aligned}
$$

These relations determine $\Sigma_{c r}^{\alpha q}(\tau)$ by means of $(4.3)$. 
${ }^{1}$ A. N. Schellekens and W. L. van Neerven, Phys. Rev. D 21, 2619 (1980).

${ }^{2}$ A. P. Contogouris and J. Kripfganz, Phys. Rev. D 20, 2295 (1979).

${ }^{3}$ H. D. Politzer, Nucl. Phys. B129, 301 (1977); D. Amati, R. Petronzio, and G. Veneziano, ibid. B140, 54 (1978); B146, 29 (1978); H. Georgi, M. Machacek, H. D. Politzer, and G. G. Ross, Phys. Lett. 78B, 281 (1978) and Nucl. Phys. B152, 285 (1979); S. Libby and G. Sterman, Phys. Lett. 78B, 618 (1978) and Phys. Rev. D 18, 3252 (1978); C. T. Sachrajda, Phys. Lett. 73B, 185 (1978).

${ }^{4}$ S. D. Drell and T. M. Yan, Phys. Rev. Lett. 25, 316 (1970) and Ann. Phys. (N.Y.) 66, 578 (1971).

${ }^{5} \mathrm{~J}$. Abad and B. Humpert, Phys. Lett. 80B, 286 (1979); J. Kubar André and F. E. Paige, Phys. Rev. D 19, 221 (1979); G. Altarelli, R. K. Ellis, and G. Martinelli, Nucl. Phys. B157, 461 (1979); A. P. Contogouris and J. Kripfganz, Phys. Rev. D 19, 2207 (1979); B. Humpert and W. L. van Neerven, Phys. Lett. 85B, 293 (1979).
${ }^{6}$ G. Altarelli and G. Parisi, Nucl. Phys. B126, 298 (1977).

${ }^{7}$ D. A. Ross and C. T. Sachrajda, Nucl. Phys. B149, 497 (1979).

${ }^{8}$ H. Strubbe, Comput. Phys. Commun. 8,1 (1974).

${ }^{9}$ G. Curci, W. Furmanski, and R. Petronzio, CERN Report No. TH-2815 (unpublished).

${ }^{10}$ Although they start from the correct anomalous dimension, the relation between the anomalous dimension and the transition function in Ref. 7 is incorrect. This can be attributed to mistakes in their formulas (3.13) and (3.15), both contributing an extra factor 2 . This explains the extra factor 4 in their formula (4.8) (compare for instance Ref. 12).

${ }^{11}$ This linear behavior is not visible in Fig. 4. Closer examination shows that the function $\Sigma_{c r}^{q q}(\tau)$ changes sign at $\tau=0.66$, which causes the result for large $\tau$ to be much smaller than the limiting behavior suggests.

${ }^{12}$ A. Buras, Rev. Mod. Phys. 52, 199 (1980).

${ }^{13} \mathrm{M}$. Chaichian, M. Hayashi, and T. Honkaranta, University of Helsinki Report No. HU-TFT-80-9 (unpublished). 